\title{
Asalto de ciudades durante la República Romana (200-167 a.C.): esclavización de supervivientes en contextos de guerra
}

\author{
José Antonio Martínez Morcillo \\ Universitat de les Illes Balears \\ josea.martinez@uib.es
}

Recibido: 1 de octubre de 2015

Aceptado: 18 de noviembre de 2015

\section{RESUMEN}

La expansión de Roma en el mundo mediterráneo motivó el sometimiento a la dicio romana de un gran número de pueblos y ciudades, un fenómeno que se concretó a varios niveles. La más violenta de estas intervenciones, la oppugnatio, llevaba implícita una serie de actuaciones que afectaban no sólo a los bienes del núcleo vencido, sino también a la libertad e integridad física de sus habitantes. En el primer tercio del siglo II a.C., y especialmente atendiendo a tres variables (desarrollo urbano, nivel de riquezas y ubicación geográfica), pretendemos reflexionar sobre la justificación legal de la esclavización de los supervivientes y, a su vez, determinar los factores que influyeron, en nuestra opinión, en la aplicación diferencial de esta represalia.

Palabras clave: República romana. Expansionismo romano. Oppugnatio. Esclavización.

\section{Assault of Cities during the Roman Republic (200-167 B.C.): Enslavement of Survivors in Contexts of War}

\begin{abstract}
The Roman Expansion in the Mediterranean World motivated the submission to the Roman dicio of a large number of populations. This phenomenon was implemented in several levels. The most violent of these interventions, the oppugnatio, implied a series of actions that affected the properties of the nucleus, but also the freedom of its inhabitants and their physical integrity. In the first third of the second century B.C., specially according to three variables (urban development, level of wealth and geographic location), we intend to ponder the legal justification for the enslavement of survivors and, at the same time, we try to determinate the factors which influenced in the differential implementation of this form of retaliation.
\end{abstract}

Keywords: Roman Republic. Roman Expansionism. Oppugnatio. Enslavement.

Sumario: 1. Introducción. 2. Las represalias tras oppugnatio. 3. La esclavización tras el asalto de ciudades. 4. La toma de prisioneros en los asaltos de campamentos. 5. Conclusiones. 


\section{Introducción ${ }^{1}$}

En el arco temporal que abarca desde el año 200 -finalización de la II Guerra Púnicahasta el 167 -victoria romana en la III Guerra Macedónica $-^{2}$ la potencia itálica va a protagonizar una intensa actividad bélica tanto en Oriente (II Guerra Macedónica, guerra contra Antíoco III y conflicto contra Perseo) como en Occidente (con el desarrollo de campañas militares en Hispania, Galia Cisalpina, Liguria e Histria). ${ }^{3}$ En este contexto, la potencia itálica vertebró su dominio a varios niveles. En primer lugar, hallamos aquellos núcleos que suscribieron acuerdos con Roma sin que mediaran acciones bélicas y que se concretaron a través de pactos de amicitia y foedera. ${ }^{4}$ Las poblaciones que no se adhirieron voluntariamente mediante estas fórmulas quedaron integradas en un segundo nivel que implicaba un alto grado de dependencia y sumisión: la deditio. Esta forma de rendición incondicional implicaba la entrega incruenta del núcleo y comportaba una suspensión temporal de garantías, durante la cual se ponía a disposición del magistrado encargado de la campaña la totalidad de los bienes públicos y privados. ${ }^{5} \mathrm{El}$ general, junto con su consilium, decidía entonces la restitutio de la ciudad, no sin antes imponer una serie de cláusulas relativamente homogéneas que se vertebraron en torno a la solicitud de rehenes, la entrega de armas y la satisfacción de multas (en numerario o en especie) en concepto de indemnizaciones de guerra ${ }^{6}$ Finalmente, los oppida que persistieron en la lucha tuvieron que hacer frente a represalias de mayor calado que se justifican mediante la resistencia presentada.

1 Este trabajo se inscribe en el ámbito del Proyecto Entre la paz y la guerra: Alianzas, confederaciones $y$ diplomacia en Occidente mediterráneo (siglos III-I a.C.) (HAR2011-27782), Dirección General de Investigación y Gestión del Plan Nacional de I+D+I, Ministerio de Economía y Competitividad, Gobierno de España.

2 En adelante, todas las fechas son a.C.

3 Sobre los conflictos mencionados vid. BAdian 1959; Harris 1979, 200-254; PeYre 1979; Gruen 1984; Richardson 1986; Foraboschi 1992, 75-102; NACo 2006; Olesti-Vila 2006; ECKSTEIN, 2008; Rosenstein 2012, 176-239; PÉREZ RuBIo et alii 2013.

4 Sobre la amicitia vid. BALdSON 1954; Gruen 1984, 54-95; SERrano 1989; Burton 2011. Con respecto a los foedera, vid. GARcía Riaza 2002, 149-162, con un estudio específico de los foedera suscritos por Q. Fabio Máximo Serviliano en Hispania Ulterior en 140 (App. Hisp. 69-70) y el del 137 entre C. Hostilio Mancino y los numantinos (App. Hisp. 80; Plut. T. G. 5).

5 Sobre la deditio y sus implicaciones vid. TAüBler 1915, 341-344; PARAdisi 1941; VISSCHER 1946; Bellini 1964; DAhlheim 1965; Flurl 1969; González Román 1990; Ziegler 1991. La deditio de Alcántara representa uno de los ejemplos paradigmáticos en el ámbito hispano, vid. LóPEz MELERo - SÁNCHEZ ABAL GARCía JimÉnEz 1984. Para recientes estudios de esta fórmula jurídica vid. GARCía Riaza 2002, Cf. GARCíA RIAZA 2011; ID. 2012, 217-218. La deditio imponía al imperium del magistrado una serie de restricciones en la toma de decisiones, de modo que era ilícito la aplicación de medidas cruentas sobre la población y el saqueo del núcleo. Para los casos específicos de incumplimiento de estas prerrogativas vid. MarTínez MorCiLlo 2011.

6 Los rehenes constituyeron la manifestación y garantía del cumplimiento de la fides, vid. AYMARD 1967; ID. 1967a; ID. 1967b; Lonis 1969; ID. 1977, 215-234; AMIT 1970; KNAPP 1977; PANAGOPOUlos 1978; Moscovich 1983; Ndiaye 1995; García Riaza 2006; Álvarez PÉrez-Sostoa 2009. Por su parte, la entrega de armas se convierte en el elemento esencial para iniciar el proceso jurídico de rendición, como se pone de manifiesto en el episodio del 182, en que los ligures se rinden a M. Claudio Marcelo, vid. Liv. 40.16.6: ... per deditionem ligures recipi, et receptis arma adimi ... Asimismo, César también se hace eco de este requisito fundamental, Caes. BG 2.32.1: Ad haec Caesar respondit: se magis consuetudine sua quam merito eorum civitatem conseruaturum, si prius quam murum aries attigisset, se dedidissent; sed deditionis nullam esse 
El saqueo de los núcleos asaltados se convirtió en la acción punitiva más extendida, mientras que en determinados casos se optó por intervenir directamente sobre los supervivientes, procediéndose de este modo a su esclavización y, en última instancia, a la aplicación de la pena de muerte. ${ }^{7}$

En el presente trabajo analizaremos los casos de oppugnationes, con la pretensión de identificar los factores que modulaban la aplicación de los diversos métodos de sumisión, en función de las dicotomías ciudad-campo y riqueza-pobreza (y averiguar, de este modo, si existió un criterio diferencial en la aplicación de represalias tras asalto armado basado en la riqueza que albergaban las ciudades sometidas), así como discernir la posible existencia de un método diferencial en función del contexto de intervención representado por el binomio Oriente-Occidente.

\section{Las represalias tras oppugnatio}

El asalto de núcleos de población como mecanismo de sumisión se hallaba legalmente justificado en casos de extrema oposición, de modo que la apropiación de los bienes de los vencidos y la esclavización de los supervivientes constituyeron represalias que, a pesar de su elevado nivel punitivo, se encontraban al amparo de la costumbre. ${ }^{8}$ Entre estas disposiciones, el saqueo se convierte en el recurso básico que constituye no sólo la represión del vencido, sino también un método de financiación de los ejércitos en campaña. ${ }^{9}$ La planificación y la toma de decisiones sobre el asalto y, también, el reparto posterior del botín correspondían al general, como se documenta, por ejemplo, en 190 durante la deditio de Focea. ${ }^{10}$ En este caso, las tropas del pretor L. Emilio Régulo desatendieron a las indicaciones de su comandante y sometieron al núcleo a pillaje. El magistrado recordó a sus soldados que sólo las ciudades asaltadas podían ser saqueadas y que, en todo caso, pertenecía al general la potestad de decidir en tal sentido. ${ }^{11}$ Asimismo, la toma de Carthago Nova por P. Cornelio Escipión en el 209 constituye una clara evidencia acerca del carácter reglado y metódico del saqueo en el que, además, las fuentes aluden a los mecanismos del posterior reparto del

condicionem nisi armis traditis. Sobre las indemnizaciones económicas vid. GARCía RiazA 1999; ID. 1999a; ID. 1999b; ID. 1999c; ÑACO - ARRAYÁs 2006.

7 Acerca de las represalias aplicadas sobre los vencidos vid. GARCía Riaza 2007; MARCo Simón 2006. En relación al saqueo vid. ZioLKOWSKi 1995.

8 El general contaba con una serie de restricciones en el ejercicio de su mandato y, como garante de la auctoritas imperatoris populi Romani (Cic. Verr. 2.16), se hallaba supervisado por el Senado, aunque en ese campo establecido por la legalidad le permitía un amplio margen de maniobra en la toma de decisiones referentes a la imposición de represalias. Acerca de los límites del imperium del magistrado romano vid. Combès 1966, 375; Álvarez Pérez-Sostoa 2009, 154; Andreu Pintado 2012.

9 En este sentido se deben tener presentes las intenciones que impulsaban a los generales a conseguir el mayor éxito posible de sus campañas, en un ambiente de rivalidad aristocrática en la que la consecución del Triunfo desempeñaba un papel fundamental, vid. HARris 1971, 1382-1383; ID. 1979, 54-105. Por otra parte, la apropiación de botín, junto a la solicitud de indemnizaciones económicas en casos de deditio, constituiría la base de lo que se ha denominado como "war economy" en los últimos años, vid. Naco 2010, 171.

10 Para un estudio del saqueo de núcleos vid. Ziolkowski 1995, cf. Martínez Morcillo 2013.

11 Liv. 37.32.12: dicendo captas, non deditas diripi urbes, et in iis tamen imperatoris, non militum arbitrium esse. 
botín. ${ }^{12}$ En el mismo sentido parecen apuntar otros casos, como la campaña dirigida por Cn. Manlio Vulsón en 189 contra los tectosagos en Galacia y la intervención de L. Emilio Paulo en 167 en el Epiro. ${ }^{13}$ Estas evidencias nos inducen a sospechar que nos hallamos ante un procedimiento planificado y regulado, aspectos que subrayan el carácter oficial de la intervención, diferenciándose del pillaje libre y descontrolado. ${ }^{14}$ Asimismo, en situaciones en que la oposición a la dominación romana fue más intensa, los generales optaron por intervenir sobre las estructuras de los núcleos sometidos y sobre su territorio. ${ }^{15}$

A un segundo grado de severidad corresponden aquellas represalias que repercutieron directamente en la integridad física de los habitantes de los núcleos asaltados. El magistrado podía ordenar la captura de los supervivientes y su posterior venta - una decisión que suele aparecer en las fuentes con la expresión sub corona-, añadiendo así un mayor correctivo por la oposición presentada a la dominación romana. ${ }^{16} \mathrm{El}$ proceso de toma de prisioneros se realizaba, comúnmente, en dos fases. Tras el fin de la batalla, se procedía a la captura de los combatientes y la detención de otros individuos que no habían participado directamente en la lucha. Es precisamente en estas circunstancias cuando los prisioneros de mayor entidad política caían en manos del vencedor, como posiblemente sucediera en Hispania con la captura de los reyes Hilerno y Corrobilo en los años 193 y 192, respectivamente. ${ }^{17}$ Del mismo modo, tras la batalla de Pidna, el cónsul L. Emilio Paulo envió a sus legados con el objetivo de

12 En efecto, se dividieron los efectivos en tres grupos: aquellos que saquearon el núcleo, quienes

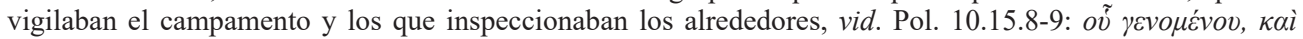

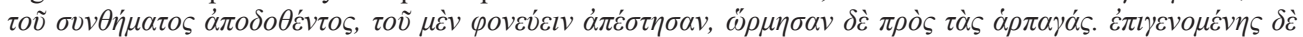

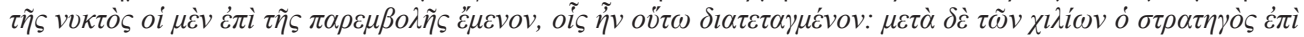

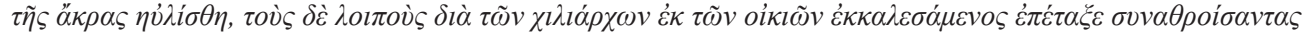

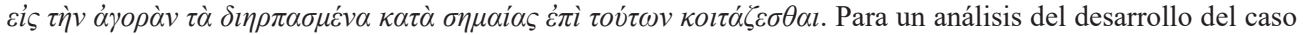
concreto de Carthago Nova y su importancia para el estudio de los saqueos vid. García RiAZA 2002, 249-250; ID. 2007, 24; ID. 2011, 55.

13 Galacia: Liv. 38.27.7-8: Romanorum pars magna ea nocte in castris hostium mansit; ceteros in sua castra consul reduxit. Postero die captivos praedamque recensuit, quae tanta fuit; SCULLARD 1973, 261; Grainger 1995, 23-42. Epiro: Liv. 45.34.1; Plut. Aem. 30, 1; Scullard 1945, 58-59. Sobre la legalidad de la intervención vid. MarTínez Morcillo 2011, 75-76; ID. 2012, 285-289, que incluyen un estudio más detallado de la campaña.

14 Existe, adicionalmente, un segundo tipo de represalia que repercute directamente sobre los bienes del bando sometido. El general podía decidir, en función de la beligerancia presentada por el vencido, intervenir sobre su territorio a través de diversas fórmulas, tales como el desplazamiento de poblaciones de la montaña al llano, la destrucción de estructuras (o del núcleo por completo), la deportación y la expulsión definitiva, vid. García Riaza 2012, 207-234. Sobre el aspecto específico de la deportación vid. Pina Polo 2004; ID. 2006.

15 Sobre este aspecto vid. Martínez Morcillo 2012a.

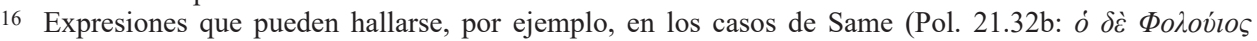

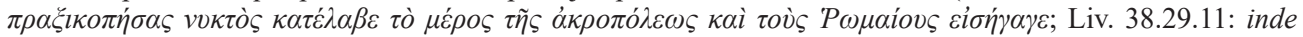
postero die dediti direpta urbe sub corona omnes venierunt), Histria (Liv. 41.11.8: Duo deinde oppida, Mutila et Faveria, vi capta et deleta. Praeda, ut in gente inopi, spe maior fuit, et omnis militibus concessa est. quinque milia capitum sescenta triginta duo sub corona venieru) y Haliarto (Liv. 42.63.10: deditione facta sub corona venierunt. Fuerunt autem duo milia ferme et quingenti).

17 Sobre la captura del rey Hilerno, que se contextualiza en la campaña del pretor M. Fulvio Nobilior contra vacceos, vettones y celtíberos, vid. Liv. 35.7.8: regem Hilernum vivum cepit. En referencia al rey Corrobilo, capturado por el propretor C. Flaminino tras la toma de Licabrum, vid. Liv. 35.22.6: nobilem regulum Corrobilonem vivum cepit. 
arrestar a dos de los promotores de la guerra. El primero, Gencio, cayó en manos de L. Anicio Galo, después de que éste forzara la deditio de varias plazas fuertes con el objetivo de interrumpir las vías de escape del rey ilirio. ${ }^{18}$ Un caso análogo sucede con Perseo, quien una vez acorralado en Anfípolis se rindió finalmente a P. Cornelio Escipión Nasica. ${ }^{19}$ Asimismo, la medida podía aplicarse en ocasiones sobre un sector específico de la población, como se puede documentar en Alce en 179, donde 320 enemigos fueron privados de su libertad durante el combate y, tras la captura del oppidum, varios nobles hispanos corrieron la misma suerte. ${ }^{20}$ Finalmente, y con la intención de añadir un correctivo aún más severo, el general podía recurrir al arresto de la totalidad de los habitantes. El destino de los prisioneros no aparece referenciado, generalmente, en el registro documental, aunque su esclavización y posterior venta seguramente constituyó uno de los procedimientos más extendidos, como trataremos más adelante.

En un tercer nivel de gravedad, el magistrado romano podía también decidir la condena a muerte de algunos sectores de la población vencida. El carácter selectivo de la medida se pone de manifiesto tras el asalto y saqueo de Antipatrea en el 200, donde el legado L. Apustio ajustició los adultos que se encontraban en edad militar (puberibusque interfectis), una decisión espoleada por la intensa resistencia presentada por el núcleo. ${ }^{21}$ También en Iliturgi en 195 M. Helvio decidió, tras el asalto del oppidum, la aniquilación de los puberes con el fin de diezmar los elementos opositores a la dominación romana. ${ }^{22}$ Análogamente, ese mismo año el cónsul M. Porcio Catón consiguió sofocar la rebelión que había iniciado la facción antirromana del núcleo de Bergio. ${ }^{23}$ En este caso, las acciones emprendidas por el general se concretaron en tres direcciones, en función del grado de participación y oposición presentado en la conflagración. En primer lugar, otorgó la libertad a quienes habían combatido conforme a los intereses de la potencia itálica, una política encaminada a asentar en el poder a las facciones afines a las directrices romanas. Los elementos restantes fueron apresados

18 La campaña desarrollada por L. Anicio viene detallada por en Liv. 44.17.4-5; 44.30.15-31.9. Liv. 44.31.9: Pulsi enim et fuga conglobati, cum ducenti amplius in ipsis faucibus portae cecidissent, tantum intulerunt terrorem, ut oratores extemplo ad praetorem mitteret Gentius Teuticum et Bellum, principes gentis, per quos indutias peteret, ut deliberare de statu rerum suarum posset. Acerca de la relación entre Gencio y Roma con anterioridad a la III Guerra Macedónica vid. GRUEN 1984, 419-423, con un estudio pormenorizado de las fuentes literarias. Sobre el papel de Gencio en la guerra, su derrota y la campaña de L. Anicio vid. Meloni 1953, 326-329, 357, 408 y 422. Posteriormente, Gencio desfiló en el Triunfo del legado, vid. Pol. 30.22.1-12; Liv. 45.16.2; 26.1-5; 43.1-9; App. Ill. 9; cf. Broughton 1951, 434.

19 Sobre el envío del legado a por Perseo vid. Liv. 44.46.1-2; Plut. Aem. 26, 4. Acerca de la rendición de Perseo vid. Pol. 29.14-15; Liv. 34.35.14-23; 36.9-14; 38.1-3; Plut. Aem. 15-18; Broughton 1951, 429.

20 Liv. 40.48.7: capti vivi trecenti viginti; Liv. 40.49.4: multi captivi nobiles in potestatem venerunt.

21 Liv. 31.27.4: armis adortus expugnauit puberibusque interfectis. Sobre el desarrollo de la campaña y la resistencia mostrada por Antipatrea vid. Liv. 31.27.3: Ac primo evocatos principes ad conloquium, ut fidei Romanorum se committerent, perlicere est conatus; Liv. 31.27.4: ... ui atque armis adortus expugnauit ...

22 Liv. 34.10.2: duodecim milia ex iis caesa, oppidum Iliturgi receptum et puberes omnes interfectos. Sobre la campaña de M. Helvio vid. Liv. 34.10.1-2: Eodem tempore M. Heluio decedenti ex ulteriore Hispania cum praesidio sex milium dato ab Ap. Claudio praetore Celtiberi agmine ingenti ad oppidum Iliturgi occurrunt. viginti milia armatorum fuisse Valerius scribit; cf. RICHARDSON 1986, 86. Asimismo vid. Alvar 2000.

23 Investigaciones recientes apuntan que se trató, en efecto, de una rebelión interna, vid. GARCÍA RIAZA 2012, 211-212. 
y vendidos como esclavos, mientras que una pequeña parte de los defensores de la ciudad, calificados por Livio como praedones, fueron acusados de ser los promotores de la revuelta y se procedió a su ejecución. ${ }^{24}$ Finalmente, en el año 177 el cónsul C. Claudio Pulcro logró someter los núcleos de Mutila y Faveria en la región de Histria, donde procedió a la decapitación de los responsables de la confrontación. ${ }^{25}$

El carácter selectivo de la pena capital y el escaso número de testimonios que las fuentes referencian responden, a nuestro entender, a motivaciones que escapan al ámbito estrictamente jurídico, de modo que debemos señalar que los intereses puramente económicos de los generales influyeron notablemente a la hora de privar de la libertad a los supervivientes de la confrontación, ya que era preferible la venta de los supervivientes a su eliminación. ${ }^{26}$ De este modo, si para el vencido constituía una medida punitiva de carácter personal, para el bando vencedor representó un mayor acceso a ingresos. ${ }^{27}$ Debemos asimismo aseverar que la gran mayoría de los núcleos asaltados carecían de grandes cantidades de objetos de valor, de modo que para paliar el déficit de botín, e incluso su ausencia, la venta de prisioneros se convirtió en un método alternativo de financiación. De hecho, en algunos casos se tuvo que recurrir a la esclavización de poblaciones como única forma de obtener ingresos. Así sucede en la toma de Numancia por parte de P. Cornelio Escipión Emiliano en 133, en que el reparto de plata entre los soldados debió realizarse a partir del beneficio obtenido con la venta de los supervivientes, ya que, como señala Apiano, resultaba improbable que en la ciudad se hallaran bienes cuya venta permitiera la obtención de grandes cantidades de numerario. ${ }^{28}$

En definitiva, podemos afirmar que si bien existían unas directrices de intervención en casos de oppugnatio - concretadas en el saqueo del núcleo rendido, la destrucción del mismo, la esclavización de sus habitantes y la modificación territorial-, la potestad recaía en última instancia en el general encargado de la campaña, de modo que éste podía decidir el grado de aplicación de las represalias en función de intereses diversos. En este sentido, no debemos despreciar la enorme influencia de las variables económicas en el proceso de toma de decisiones.

24 Liv. 34.21.5-6: huius potitus loci consul eos qui arcem tenuerant liberos esse cum cognatis suaque habere iussit, Bergistanos ceteros quaestori ut venderet imperavit, de praedonibus supplicium sumpsit. Para un estudio de la campaña de M. Porcio Catón vid. Astin 1978; Richardson 1986, 83 y ss.; MARTínez GÁZQUEZ 1992.

25 Liv. 41.11.8: auctores belli virgis caesi et securi percussi. Sobre Histria la historiografía ha centrado su atención, principalmente, en el conflicto que Roma tuvo que hacer frente en los años 221-220. En estos momentos, la piratería histria suponía un grave problema para el expansionismo romano, que según Dell concibió la guerra como un apéndice de su expansión por Galia Cisalpina. Para un estudio de este conflicto vid. Pol. 21.16.4; Liv. 10.2.4; App. Ill. 8; DeLl 1967, 351-352; ID. 1970.

26 Vid. tablas del Anexo.

27 TAÜBler 1915, 81-83; Volkmann 1961, 46-50; GARrido-Hory 1996; Nicosia 1996, 145-151; NaCo 2003,145-151; ID. 2005; GARCía RiAZa 2007, 25.

28 App. Hisp, 96, menciona que los numantinos tuvieron que recurrir a lamer las pieles de los animales e, incluso, a la antropofagia, una situación que refleja la precariedad en que se hallaba el núcleo durante el asedio romano. La venta de los supervivientes se referencia en App. Hisp. 97, tras elegir Escipión 50 para su desfile triunfal; vid. García Riaza 2007, 25. 


\section{La esclavización tras el asalto de ciudades}

El análisis de los casos de poblaciones sometidas, aún teniendo en cuenta el carácter fragmentario del registro documental, permite inferir la existencia de una serie de factores diferenciales en la aplicación de la esclavitud como represalia. ${ }^{29}$ Del conjunto de oppugnationes que Roma finalizó en contextos de conflicto, observamos una clara asimetría territorial, de modo que de los 19 casos detectados en Oriente durante el período de estudio tan sólo 3 culminaron con la captura de efectivos humanos (vid. Tabla 1a); en Occidente, por su parte, la proporción es mucho mayor, ya que de los 12 asaltos documentados, 8 culminaron con la esclavización de los supervivientes (vid. Tabla 1b).

En todos los episodios relativos a los conflictos de Roma con el mundo helenístico -a excepción de la campaña de L. Emilio Paulo- la dureza de las decisiones se justifica legalmente por la oposición presentada. Así se pone de manifiesto en el asedio de Haliarto (Beocia), que fue iniciado por P. Cornelio Léntulo y continuado en 171 por el pretor de la flota C. Lucrecio Galo. ${ }^{30}$ En esta ocasión es evidente que la prolongada resistencia del núcleo influyó notablemente en el castigo impuesto tras la oppugnatio, un hecho que explicaría la inmediata venta de 2.500 habitantes, el saqueo de la ciudad y su demolición. ${ }^{31}$ Un caso análogo se puede atestiguar en la toma de Same en 189, que decidió persistir en su oposición a pesar del avance de las tropas de M. Fulvio Nobilior en Cefalania. Esta actitud ofreció al general romano la justificación para iniciar el asedio del núcleo, que fue saqueado y sus habitantes esclavizados. ${ }^{32}$

Por su parte, en Occidente el criterio de responsabilidad política tan sólo puede detectarse en algunos episodios. De esta forma sucede en la toma de Bergio en 195 -un testimonio que ya hemos analizado-, en que la facción antirromana había depuesto

29 Las fuentes literarias focalizan mayoritariamente su atención en el arresto de personajes de mayor renombre ya que, como queda reflejado en los desfiles triunfales, son la prueba viva de la victoria romana. Sin embargo, estos individuos representan tan sólo un ínfimo porcentaje del total de prisioneros conseguidos por la potencia itálica. Es evidente que la situación de los prisioneros de mayor entidad era diferente de aquellos que pertenecían a niveles sociales inferiores. Aún con las quejas de Orosio sobre la suerte que corrieron varios monarcas que cayeron en manos de Roma (Oros. 5.1.9: omitto de regibus magnarum opum, magnarum virium, magnae gloriae, diu potentissimis, aliquando captis, serviliter catenatis, sub iugum missis, ante currum actis, in carcere trucidatis), el destino de éstos solía ser mejor que el del resto de apresados; vid. Álvarez PÉrezSostoa 2009, 170. Sobre el papel de estos personajes en los Triunfos vid. BASTIEN 2007, 225-237; BEARD 2009, 147-190. Acerca del Triunfo en general vid. Versnel 1970; Auliard 2001.

30 Liv. 42.63.3, la ciudad de Haliarto había mantenido su filiación a la causa macedónica desde la ascensión de Perseo, junto a Coronea y Tisbe y el resto de la Confederación Beocia, vid. MelONI 1953,147 y 199-200. Acerca de P. Cornelio Léntulo vid. Liv. 42.47.12; 42.56.3; Broughton 1951, 417, Cornelio Léntulo desempeñó la labor de tribuno bajo las órdenes del cónsul P. Licinio Craso (Liv. 42.49.9), que fue el embajador enviado a Beocia.

31 Liv. 42.63.10-11: ... cum spei nihil superesset, deditione facta sub corona venierunt. fuerunt autem duo milia ferme et quingenti; Liv. 42.63.11: Ornamenta urbis, statuae et tabulae pictae, et quidquid pretiosae praedae fuit, ad naves delatum; urbs diruta a fundamentis. Gran parte de las obras de arte que formaban parte del botín fueron utilizadas para decorar el santuario de Asclepio, vid. Liv. 43.4.7; 43.7.10. Sobre el saqueo de obras de arte y su uso posterior vid. Gruen 1984, 258-261. Para un estudio completo del ataque sobre Haliarto y sus consecuencias vid. Meloni 1953, 243-263.

32 Liv. 38.29.11: Samaei postquam captam partem urbis ab hostibus senserunt, cum coniugibus ac liberis in maiorem refugerunt arcem. Inde postero die dediti direpta urbe sub corona omnes venierunt. 
a su dirigente, quien solicitó el auxilio de M. Porcio Catón. La plaza fue finalmente ocupada y su gobierno filorromano repuesto. Como represalia se procedió al arresto (y presumiblemente la esclavización) de quienes habían participado contra el bando romano, mientras que aquellos que habían incitado a la revuelta fueron condenados a muerte. ${ }^{33}$ De nuevo en el año 184 , el pretor A. Terencio Varrón procedió de manera análoga contra la revuelta de los suesetanos, que culminó con la toma de la ciudad de Corbio, un hecho que según Salinas vino motivado por las fortificaciones que se estaban iniciando en la zona y provocó, en consecuencia, la venta de los habitantes del núcleo. ${ }^{34}$ Ya en el 179, Ti. Sempronio Graco logró asaltar Alce, que había resistido las acometidas del ejército del propretor. Éste ordenó el saqueo del núcleo e hizo prisioneros a varios individuos que formaban parte del sector dirigente del mismo. ${ }^{35}$ Un número importante de efectivos habían sido capturados en la batalla que precedió al avance hacia la ciudad. ${ }^{36}$ Un último es el de Histria, donde en el año 178 el rey Epulón había logrado formar un gran ejército con la colaboración del régulo galo Catmelo. ${ }^{37}$ Este hecho provocó las suspicacias del Senado, que decidió destinar allí a los cónsules A. Manlio Vulsón y M. Junio Bruto. La inestabilidad en la zona continuó al año siguiente, de modo que los patres enviaron también al cónsul C. Claudio Pulcro para que prestase auxilio a los generales del año anterior. La guerra finalizó con la captura de los núcleos de Mutila y Faveria, que fueron saqueados y donde se apresó a un total de 5.732 histrios. ${ }^{38}$

Sin embargo, en algunos contextos el grado de oposición presentado no se corresponde con las represalias aplicadas. En el escenario oriental, el episodio más claro es la campaña de L. Emilio Paulo contra las ciudades del Epiro en el 167. La actuación de Paulo se vio impulsada por la decisión senatorial de ofrecer como botín para el ejército del procónsul las 70 ciudades molosas, ${ }^{39}$ una intervención que constituye un flagrante incumplimiento del ius belli, ya que la totalidad de las plazas se encontraba bajo el dominio romano y sometidas a la vigilancia de las guarniciones que fueron

33 Liv. 34.21.5-6: huius potitus loci consul eos qui arcem tenuerant liberos esse cum cognatis suaque habere iussit, Bergistanos ceteros quaestori ut venderet imperavit, de praedonibus supplicium sumpsit. AstiN 1978, 304-305; RICHARDSON 1986, 83.

34 Salinas 1986, 11; ID. 1989, 71, vid. Liv. 39.42.1: in citeriore A. Terentius in Suessetanis oppidum Corbionem vineis et operibus expugnauit, captiuos vendidit.

35 Liv. 40.49.4-5: postremo et inde praemissis oratoribus in dicionem se suaque omnia Romanis permiserunt. magna inde praeda facta est. multi captivi nobiles in potestatem venerunt, inter quos et Thurri filii duo et filia. regulus hic earum gentium erat; cf. Fernández CANosa 1988; Ñaco 2003, 159.

36 Liv. 40.48.7: eo die novem milia hostium caesa; capti vivi trecenti viginti, equi centum duodecim, signa militaria triginta septem.

37 Liv. 41.1.1: a patre in pace habitam armasse eoque iuventuti praedandi cupidae pergratus esse dicebatur. consilium de Histrico bello cum haberet consul, alii gerendum extemplo, antequam contrahere copias hostes possent, alii consulendum prius senatum censebant; Liv. 41.1.8: ab eadem regione mille ferme passuum castra erant Gallorum: Catmelus regulus praeerat tribus haud amplius milibus armatorum.

38 Liv. 41.11.8: Duo deinde oppida, Mutila et Faveria, ui capta et deleta. Praeda, ut in gente inopi, spe maior fuit, et omnis militibus concessa est. quinque milia capitum sescenta triginta duo sub corona venierunt.

39 Liv. 45.34.1; Plut. Aem. 30.1. Acerca de la situación del Epiro vid. Pol. 30.15; Str. 7.7.3; SCULLARD 1945. Para un estudio de la campaña de L. Emilio Paulo vid. Martínez Morcillo 2012, 283-287. 
destinadas por el propretor L. Anicio Galo. ${ }^{40}$ De este modo, en el recorrido de regreso a Roma, el grueso del ejército consular se detuvo en el Epiro, donde exigió a cada ciudad que depositase extramuros sus fondos de metal precioso en un día y una hora determinados, a cambio de retirar los efectivos itálicos que se hallaban en su interior (missis centurionibus in singulas urbes, qui se dicerent ad praesidia deducenda venisse, ut liberi Epirotae sicut Macedones essent). ${ }^{41}$ Según Livio y Plutarco, el procónsul envió con antelación a cada núcleo un destacamento con el fin de que estuvieran todos ellos situados al unísono en el momento acordado. ${ }^{42}$ De este modo, todos los núcleos fueron saqueados, se derruyeron sus murallas y se hicieron en total 150.000 prisioneros. $^{43}$

En cuanto a las poblaciones occidentales, en la mayor parte de los asaltos el grado de oposición presentado por éstas no queda reflejado con claridad en el registro documental. Esta anomalía denota, a nuestro entender, una intencionalidad por parte de nuestras fuentes de subrayar el amplio abanico de posibilidades que poseían los magistrados encargados de las diferentes campañas para determinar el alcance de las represalias. Asimismo, se colige que, al contrario de como sucedió en Oriente, la intervención de los generales se caracterizó por un mayor grado de laxitud en la aplicación de medidas punitivas. En el caso occidental, el asalto y el saqueo de núcleos de población no se ordenó como recurso extremo ante colectivos refractarios a la rendición, sino en calidad de represalia política, como queda reflejado en Liguria con la campaña que en 192 lideró el cónsul L. Quincio Flaminino. El detonante de la intervención fue el envío a Roma de una misiva en 193, en la que los dirigentes de Pisa informaban acerca de una conspiración de los ligures contra Roma (coniuratione per omnia conciliabula universiae gentis facta). ${ }^{44}$ Estos pueblos -cuyo nombre desconocemos debido a la imprecisión de Livio- habían apresado a varios aliados y ciudadanos romanos, un hecho que impulsó a Flaminino a devastar el territorio y a capturar seis plazas fuertes, que fueron saqueadas. Se procedió también a la captura de los supervivientes, aunque las fuentes no revelan la cantidad total de individuos que fueron arrestados. ${ }^{45}$ Sin embargo, la zona no quedó pacificada hasta el año 185 , cuando el cónsul Ap. Claudio Pulcro asaltó seis plazas de los ingaunos, tomó un gran

40 Liv. 45.26.4. Un método utilizado primordialmente para descargar el coste del mantenimiento del ejército sobre el vencido, ya que las ciudades tenían la obligación de mantener los efectivos destinados tras sus murallas; vid. ÑACO 2001.

41 Liv. 45.34.1.

42 Liv. 45.34.4; Plut. Aem. 29.4.

43 Liv. 45.34.5: centum quinquaginta milia capitum humanorum abducerentur; Plut. Aem. 29.3:

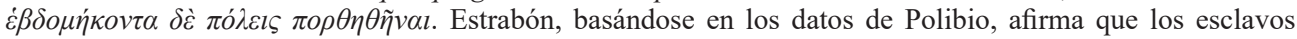

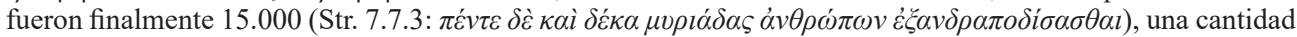
que consideramos reducida en exceso por el volumen de núcleos sometidos.

44 Liv. 34.56.2-3; cf. Lamboglia 1932, 11-12; Dyson 1985, 98. Sobre la campaña de Termo vid. Broughton 1951, 346 y 351. Solicitó el Triunfo en el 190, pero fue rechazado por el Senado. Asimismo vid. Liv. 37.46.1-2; BONNEFOND-COUDRY 1989, 271.

45 Liv. 35.40.4: Ab altero consule ager Ligurum late est vastatus castellaque aliquot capta, unde non praeda modo omnis generis cum captivis parta sed recepti quoque aliquot cives sociique qui in hostium potestate fuerant. 
número de prisioneros y condenó a muerte a 43 individuos acusados de ser responsables de la confrontación. ${ }^{46}$

\section{La toma de prisioneros en los asaltos de campamentos}

Además del asalto a núcleos fortificados, hallamos un segundo nivel de confrontaciones: las batallas en contextos no urbanos. Este tipo de actuaciones se desarrollaron principalmente en el ámbito de Cisalpina, Liguria, Hispania y Galacia. Tras la victoria, el saqueo de los campamentos y la captura de los supervivientes se convirtieron en medidas punitivas habituales, que permitirían el acceso a las reservas logísticas del ejército rival, redondeando los beneficios de la campaña con la apropiación de los bienes de los soldados enemigos y la esclavización de los prisioneros obtenidos en el propio campamento.

Uno de los episodios mejor conocidos es el del año 189, que enfrentó a las tropas romanas contra los gálatas - quienes habían colaborado activamente con Antíoco III- en un contexto no urbano. ${ }^{47}$ Los tolostobogios fueron atacados en primer lugar y sufrieron el asalto y saqueo de su campamento por parte del cónsul Cn. Manlio Vulsón. ${ }^{48}$ En cuanto al número de prisioneros, Livio afirma desconocer el total de arrestados, debido a la divergencia existente en las fuentes que utiliza para la elaboración de su trabajo. Así, Claudio Cuadrigario asegura que fueron 40.000, frente a los 10.000 que afirma Valerio Antias. ${ }^{49}$ Sea como fuere, se hizo efectiva su venta en el teatro de operaciones por la dificultad que entrañaba su traslado, y el beneficio obtenido se dividió en dos partes: la primera fue destinada al erario, mientras que el resto se distribuyó en lotes equitativos entre las tropas (cum cura, ut quam aequissima esset). ${ }^{50}$ Tras esta intervención, los tectosagos (que contaban con algunos efectivos de origen trocmo) iniciaron un proceso de negociación con el cónsul para presentar su rendición. ${ }^{51}$ Sin embargo, se trataba de una estratagema ideada para organizar sus fuerzas y emprender el ataque contra las tropas romanas, aunque el resultado no fue

46 Liv. 39.32.4: Sex praeterea oppida eorum expugnauit; multa milia hominum in iis cepit; belli auctores tres et quadraginta securi percussit; cf. Dyson 1985, 102.

47 Para una descripción de la migración y de la situación de los gálatas vid. Liv. 38.16.1-15. Tolostobogios y tectosagos habían ofrecido ayuda a Antíoco III durante el conflicto. "Atrajo a los gálatas a una alianza con regalos y con la amenaza de sus efectivos, pues consideraba que serían buenos combatientes a causa de su corpulencia" (App. Syr. 6). Grainger $(2002,322)$ contabiliza en el ejército de Antíoco III un total de 3.000 combatientes gálatas, que probablemente formarían parte de la infantería.

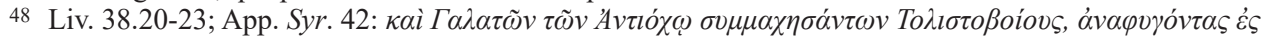

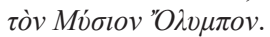

49 Liv. 38.23.8: Claudius, qui bis pugnatum in Olympo monte scribit, ad quadraginta milia hominum auctor est caesa, Valerius Antias, qui magis immodicus in numero augendo esse solet, non plus decem milia.

50 Liv. 38.23.10: Consul armis hostium uno concrematis cumulo ceteram praedam conferre omnis iussit, et aut vendidit, quod eius in publicum redigendum erat, aut cum cura, ut quam aequissima esset, per milites divisit. Sobre el procedimiento de reparto del botín vid. Shatzman 1972; ChurChill 1999; Coudry 2009, 26; TARPIN 2009, 89-90. La actuación en Galacia contra los pueblos que allí habitaban difícilmente puede desligarse del deseo de conseguir beneficio económico, vid. Heuss 1971, 110; Harris 1979, $223-225$.

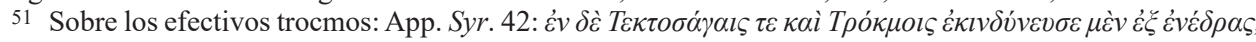

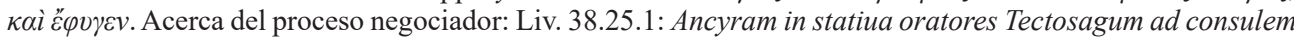


favorable a los intereses gálatas, ya que $\mathrm{Cn}$. Manlio logró asaltar el campamento, saquearlo y arrestar 8.000 enemigos, que fueron vendidos junto con el resto del botín. ${ }^{52}$

Considerando la intervención romana en Occidente, el número de casos que hemos podido documentar es mayor en comparación con el mundo helenístico. El primero de ellos corresponde al ámbito de Cisalpina, concretamente a la campaña del cónsul C. Cornelio Cetego en 197, quien se dedicó a interrumpir las vías de comunicación entre boyos e insubres, poblaciones que se habían coaligado contra Roma. La intervención del cónsul se concretó en tres direcciones. En primer lugar, tras un proceso de negociación con los cenomanos, consiguió su compromiso de que no ofrecerían ayuda militar a sus aliados y colaborarían con el cónsul en caso de que fuera necesario. ${ }^{53}$ En segundo lugar, al verse privados de sus aliados, los boyos decidieron someterse mediante deditio. Finalmente, atacó a los insubres en su territorio, consiguiendo una gran cantidad de botín y 5.200 prisioneros, entre los que se hallaba un cierto Amílcar. ${ }^{54}$

En el ámbito hispano se pueden rastrear también varios casos análogos, como en la campaña del 193, año en que el pretor P. Cornelio Escipión Nasica atacó las posiciones de los lusitanos, quienes habían logrado realizar con éxito algunas incursiones en la Ulterior. ${ }^{55}$ El magistrado tomó el campamento enemigo, capturando el botín que los lusitanos habían conseguido con sus correrías y a 540 de los combatientes rivales. Los objetos recuperados fueron transportados hasta Ilipa, a fin de que sus legítimos dueños pudieran reclamarlos. Posteriormente, la praeda sobrante -presumiblemente junto con los prisioneros- se puso a disposición del cuestor, quien procedió a su venta y al reparto del beneficio. ${ }^{56}$ En el 189 hallamos un caso análogo y, de nuevo, la cam-

uenerunt petentes, ne ante [ab Ancyra] castra moueret, quam collocutus cum suis regibus esset: nullas condiciones pacis iis non bello fore potiores.

52 Liv. 38.27.7: Postero die captivos praedamque recensuit, quae tanta fuit, quantam auidissima rapiendi gens, cum cis montem Taurum omnia armis per multos annos tenuisset, coaceruare potuit. Sobre este aspecto vid. GRAINGER 1995, 37-38.

53 Liv. 32.30.7-8: Excitis ad se principibus id agere ac moliri coepit ut desciscerent ab Insubribus Cenomani et sublatis signis aut domos redirent aut ad Romanos transirent. Et id quidem impetrari nequiit: in id fides data consuli est ut in acie aut quiescerent aut, si qua etiam occasio fuisset, adiuvarent Romanos.

54 Liv. 32.30.11-12: Quidam et a Cenomanis terga repente in ipso certamine adgressis tumultum ancipitem iniectum auctores sunt caesaque in medio quinque et triginta milia hostium, quinque milia et ducentos vivos captos, in iis Hamilcarem Poenorum imperatorem, qui belli causa fuisset; signa militaria centum triginta et carpenta supra ducenta. Resulta especialmente confusa la mención al caudillo cartaginés Amílcar. Livio confirma su muerte en la campaña de L. Furio Purpúreo en el año 200 (Liv. 31.21.18). El estudio realizado por parte de Briscoe en relación a este hecho ha servido para poner en duda la veracidad de la narración del Patavino, que al parecer en varios fragmentos de los libros XXXI a XXXIII no utiliza a Polibio como fuente principal, exponiendo una serie de casos en que se pueden hallar confusiones evidentes. Así sucede, por ejemplo, en la confusión entre $\mathrm{Cn}$. Cornelio Léntulo y $\mathrm{Cn}$. Cornelio Blasio, que son tratados como si de una misma persona se tratara en el desarrollo de la magistratura en Hispania en el año 200 (Liv. 31.50 .11 y Liv. 33.27.1). Un segundo caso se establece en el 198, en el que otorga a M. Sergio Silo el cargo de pretor urbano y peregrino en dos referencias diferentes (Liv. 32.28.2 y Liv. 33.21.9). Sobre la problemática referente a Amílcar, vid. BRISCOE 2009, 4-5.

55 BROUGHTON 1951, 348.

56 Acerca de la captura del campamento: Liv. 35.1.10: capti quingenti quadraginta, omnes ferme equites, et signa militaria capta centum triginta quattuor; de exercitu Romano septuaginta et tres amissi. Sobre el proceso de reparto: Liv. 35.1.11-12: eo victorem opulentum praeda exercitum P. Cornelius reduxit. ea omnis 
paña se dirigió contra los lusitanos, esta vez bajo el mando de L. Emilio Paulo. Tras dos años sin resultados positivos, el pretor decidió atacar el campamento enemigo, donde consiguió capturar un total de 2.300 prisioneros sin que existan datos relativos al saqueo. ${ }^{57}$ Tres años más tarde, en 186, el propretor L. Manlio Acidino Fulviano derrotó a un contingente de celtíberos en las cercanías de Calagurris, que no se vio afectada por la confrontación. Las tropas romanas se hicieron con el control del campamento tras haber abatido a 12.000 enemigos en el campo de batalla, una acción que culminó con el saqueo y el arresto de 2.000 individuos. ${ }^{58} \mathrm{La}$ comparación de las riquezas aportadas por L. Manlio en su ovatio -que ascendían en total a 52 coronas, 132 libras de oro y 16.000 libras de plata- nos hace sospechar que tales cantidades debieron proceder de la venta de los efectivos capturados. ${ }^{59}$

Centrándonos en los episodios de Liguria del 177, C. Claudio Pulcro, tras haber solventado la campaña de Histria el año anterior, se dirigió a la citada región por orden senatorial para socorrer al procónsul Ti. Claudio Nerón, que se hallaba amenazado en Pisa. La intervención de C. Claudio culminó con la captura y saqueo de la base enemiga y el arresto de más de 700 combatientes ligures. ${ }^{60} \mathrm{La}$ valoración económica de la campaña del cónsul resulta difícil de precisar, ya que los únicos datos disponibles acerca del beneficio que pudo obtener proceden de la descripción de su Triunfo. La dificultad principal estriba en que, además de los problemas que conlleva la cuantificación que ofrece Livio, ${ }^{61}$ los datos que aparecen mencionados en las fuentes (307.000 denarios y 85.702 victoriados) no proceden de una única campaña, de modo que no nos hallamos en disposición de desglosar ambas intervenciones en el estado de la investigación actual. ${ }^{62}$

ante urbem exposita est potestasque dominis suas res cognoscendi facta est; cetera vendenda quaestori data; quod inde refectum est, militi divisum. Asimismo, vid. RichardSON 1986, 96.

57 Liv. 37.57.5-6: fusi fugatique hostes; caesa decem octo milia armatorum; duo milia trecenti capti et castra expugnata. Por su parte, Plutarco (Aem. 4.3) afirma que se rindieron 250 ciudades durante la marcha de Emilio Paulo, aunque la cifra no parece ajustarse a la realidad hispana, ya que tal como afirma BRISCOE $(2003,363)$ el biógrafo convirtió en victoria la derrota sufrida por el pretor contra los lusitanos, de modo que nos hallamos ante un caso de manipulación de los acontecimientos. Acerca de la campaña vid. Martínez MorciLlo 2012, 276-280.

58 Liv. 39.21.9: ad duodecim milia hominum caesa, plus duo capta, et castris Romanus potitur.

59 Liv. 39.29.6: tulit coronas aureas quinquaginta duas, auri praeterea pondo centum triginta duo, argenti sedecim milia trecenta. El Senado no halló condiciones suficientes para otorgarle el Triunfo, ya que no había licenciado a sus tropas y no había logrado pacificar íntegramente la provincia, de modo que decidió premiarle con la ovatio, Liv. 39.29.5: exemplum obstabat, quod ita comparatum more maiorum erat, ne quis, qui exercitum non deportasset, triumpharet, nisi perdomitam pacatamque provinciam tradidisset successori. medius tamen honos Manlio habitus, ut ovans urbem iniret; ÑACO 2003, 153. Sobre el trato del botín y su procedencia vid. Shatzman 1972; Churchill 1999, 85-116; TARPin 2009, 89-90. Acerca del Triunfo y su relación con el botín vid. BASTIEN 2007, 324-331.

60 Liv. 41.12.8-9: Ad Scultennam flumen in campos progressi castra habebant hostes, ibi cum iis acie dimicatum. quindecim milia caesa, plus septingenti aut in proelio aut in castris ++ nam ea quoque expugnata sunt ++ capti, et signa militaria unum et quinquaginta capta. Ligures, reliquiae caedis, in montes refugerunt, passimque populanti campestris agros consuli nulla usquam apparuerunt arma.

61 Especialmente si atendemos a la transmisión de la información, cuyo principal hándicap radica en la cuantificación de los montantes en una unidad de cuenta que no se corresponde con el contexto descrito. Para un estudio al respecto vid. CRAWFORD 1974 II, 631; Briscoe 2008, 536; García RiazA 2011, 49.

62 Liv. 41.13.7-8: tulit in eo triumpho denarium trecenta septem milia et uictoriatum octoginta quinque milia septingentos duos. militibus in singulos quini deni denarii dati, duplex centurioni, triplex equiti. sociis dimidio minus quam civibus datum. itaque taciti, ut iratos esse sentires, secuti sunt currum. 


\section{Conclusiones}

El registro documental que hemos analizado permite identificar las características de la toma de prisioneros como represalia en contextos de oppugnatio y, asimismo, demostrar que existieron diferencias en la aplicación de represalias en los diferentes ámbitos que hemos definido. Sin embargo, esta aplicación diferencial en función del territorio no supuso una quiebra de las bases del ius belli, ya que en todo caso las medidas aplicadas se hallaban dentro de la legalidad y justificadas bien por el grado de oposición a la dominación romana, bien por las estrategias territoriales que la potencia itálica quería aplicar en cada contexto.

A pesar de que en un primer momento se pueda detectar una aplicación diferencial de represalias entre el escenario occidental y el oriental, un análisis más profundo permite descubrir la existencia de otros factores. En primer lugar, el nivel de desarrollo urbano desempeñó un papel fundamental, ya que las medidas tras las diversas oppugnationes se realizaron, mayoritariamente, en función del grado de responsabilidad política, un aspecto que se pone de manifiesto en Oriente, pero que en algunos ámbitos occidentales (como en Bergio o Alce) hemos demostrado que también existió. Por su parte, las campañas desarrolladas en campo abierto concluyeron en el saqueo de los bienes de los vencidos y en la esclavización de los combatientes, circunstancia que se cumple en ambas vertientes del Mediterráneo.

En este punto, el nivel de riqueza de las poblaciones sometidas se convirtió también en un factor a tener en cuenta. Por tanto, más allá de los aspectos tácticos o legales, y sin menoscabo de su influencia en la toma de decisiones que justificaban una intervención severa sobre aquellas poblaciones más beligerantes, intervinieron también notablemente criterios económicos. La aprehensión de los bienes del vencido, que constituirían el botín de guerra, es la represalia que en mayor medida se aplicó en las ciudades recién sometidas. Sin embargo, en aquellos núcleos cuyos recursos no alcanzaban a cubrir las expectativas de los generales (como en el caso de Numancia) y en los campamentos asaltados, la esclavización de los prisioneros se convirtió en una represalia generalizada y, a grandes rasgos, se detecta un desequilibrio entre las referencias de ambas vertientes mediterráneas, con una mayor presencia de la medida en Occidente.

El mismo proceso es detectable en el caso de confrontaciones en ambientes rurales, donde el botín aprehendido en los campamentos era generalmente inferior en comparación con las riquezas obtenidas en contextos urbanos. Así sucede, como hemos podido comprobar, en la campaña contra los tolostobogios en 189, quienes tras haber sido apresados fueron vendidos en el mismo teatro de operaciones por las dificultades que implicaba el desplazamiento de grandes contingentes humanos y, también, por la escasez de otras formas alternativas de botín. De este modo, en territorios con recursos más limitados la esclavización de prisioneros constituyó un medio de financiación militar no desdeñable. 
Tabla 1. Represalias tras oppugnatio (200-167).

1a: Oriente

\begin{tabular}{|c|c|c|c|c|c|}
\hline Año & Ciudad/Población & Ámbito & Saqueo & Prisioneros & $\begin{array}{l}\text { Pena de } \\
\text { muerte }\end{array}$ \\
\hline 200 & Antipatrea & Macedonia & Liv. 31.27 .4 & & $\begin{array}{l}\text { Liv. } \\
31.27 .4\end{array}$ \\
\hline 199 & Pelión & Macedonia & Liv. 31.40 .4 & & \\
\hline 198 & Faloria & Macedonia & Liv. 32.15.4 & & \\
\hline 198 & Elacia & Macedonia & Liv. 32.24.7 & & \\
\hline 198 & Eretria & Grecia (Eubea) & Liv. 32.16 .17 & & \\
\hline 191 & Heraclea & Macedonia & Liv. 36.24.6-7 & & \\
\hline 190 & Lamia & Grecia & Liv. 37.5.3 & & \\
\hline 190 & Focea & Asia Menor & Liv. 37.32.12 & & \\
\hline 189 & Same & Grecia & Liv. 38.29 .11 & $\begin{array}{l}\text { Liv. } 38.29 .11 \\
\text { Pol. 21.32b }\end{array}$ & \\
\hline 189 & Lago & Asia Menor & Liv. 38.15 .3 & & \\
\hline 189 & Cormasa / Círmasa & Asia Menor & Liv. 38.15 .14 & & \\
\hline 189 & Darsa & Asia Menor & Liv. 38.15 .14 & & \\
\hline 171 & Haliarto & Grecia & Liv. 42.63 .11 & Liv. 42.63 .10 & \\
\hline 171 & Malea & Macedonia & Liv. 42.67.7 & & \\
\hline 168 & Pidna & Macedonia & Liv. 44.45 .7 & & \\
\hline 168 & Melibea & Macedonia & Liv. 44.46 .3 & & \\
\hline 168 & $\begin{array}{l}\text { Eginio } \\
\text { Agasas }\end{array}$ & Grecia & Liv. 45.27.1 & & \\
\hline 168 & enios & Grecia & Liv. 45.27.4 & & \\
\hline 167 & Ciudades del Epiro & Grecia & Liv. 45.34 .4 & $\begin{array}{r}\text { Liv. } 45.34 .5 \\
\text { Plut. Aem. } 29.3\end{array}$ & \\
\hline
\end{tabular}

1b: Occidente

\begin{tabular}{|c|c|c|c|c|c|}
\hline Año & Ciudad/Población & Ámbito & Saqueo & Prisioneros & $\begin{array}{c}\text { Pena de } \\
\text { muerte }\end{array}$ \\
\hline 196 & comenses & Galia Cisalpina & Liv. 33.36.13-14 & & \\
\hline 195 & Iliturgi & Hispania & & & $\begin{array}{l}\text { Liv. } \\
34.10 .2\end{array}$ \\
\hline 195 & Bergio & Hispania & & Liv. 34.21 .5 & $\begin{array}{l}\text { Liv. } \\
34.21 .6\end{array}$ \\
\hline 193 & $\begin{array}{l}\text { vacceos } \\
\text { vettones } \\
\text { celtíberos }\end{array}$ & Hispania & & Liv. 35.7 .8 & \\
\hline 192 & Licabrum & Hispania & & Liv. 35.22.5-6 & \\
\hline 192 & ligures & Liguria & Liv. 35.21.9-11 & & \\
\hline 192 & ligures & Liguria & Liv. 35.40 .4 & Liv. 35.40 .4 & \\
\hline 185 & ingaunos & Liguria & & Liv. 39.32.4 & $\begin{array}{l}\text { Liv. } \\
39.32 .4\end{array}$ \\
\hline 184 & Corbio & Hispania & & Liv. 39.42.1 & \\
\hline 182 & Urbicna & Hispania & Liv. 40.16 .9 & & \\
\hline
\end{tabular}




\begin{tabular}{|r|l|l|l|l|l|}
\hline 179 & Alce & Hispania & Liv. 40.49.5 & $\begin{array}{l}\text { Liv. 40.48.7 } \\
\text { Liv. 40.49.4 }\end{array}$ & \\
\cline { 1 - 4 } 177 & histrios & Histria & Liv. 41.11.8 & Liv. 41.11.8 & \\
\hline
\end{tabular}

Tabla 2. Intervenciones en campo abierto (campamentos) (200-167).

2a: Oriente

\begin{tabular}{|c|c|c|c|c|}
\hline Año & Ciudad/Población & Ámbito & Saqueo & Prisioneros \\
\hline 189 & tolostobogios & Galatia & Liv. 38.23.10 & Liv. 38.23 .9 \\
\hline 189 & tectosagos & Galatia & Liv. 38.27 .7 & Liv. 38.27 .7 \\
\hline
\end{tabular}

2b: Occidente

\begin{tabular}{|c|l|c|c|c|}
\hline Año & Ciudad/Población & Ámbito & Saqueo & Prisioneros \\
\hline 197 & insubres & Galia Cisalpina & Liv. $32.30 .11-12$ & Liv. 32.30.11-12 \\
\hline 193 & lusitanos & Liguria & Liv. 35.1 .10 & Liv. 35.1 .10 \\
\hline 189 & lusitanos & Liguria & Liv. 37.57 .6 & Liv. 37.57 .6 \\
\hline 186 & celtíberos & Hispania & Liv. 39.21 .9 & Liv. 39.21 .9 \\
\hline 177 & ligures & Liguria & Liv. 41.12 .8 & Liv. $41.12 .7-9$ \\
\hline
\end{tabular}

\section{Bibliografía}

Alvar, J. (2000): "El sexo y la edad en la derrota: los romanos en Hispania", [en] M. M. Myro - J. M. Casillas - J. Alvar - D. Plácido (eds.), Las edades de la dependencia, Madrid, 363-384.

Álvarez PÉrez-SostoA, D. (2009): “El confinamiento de los prisioneros de guerra y rehenes en la Roma republicana", Veleia 26, 153-171.

Амiт, M. (1970): “Hostages in Ancient Greece”, Riv. Fil. 98, 129-147.

Andreu Pintado, J. (2012): "Imperium del magistrado provincial: límites administrativos, réditos políticos, conquistas territoriales. El caso hispano", [en] Santos Yanguas - Cruz Andreotti (eds.), 2012, 29-54.

Astin, A. E. (1978): Cato the Censor, Oxford.

Auliard, C. (2001): Victoires et Triomphes à Rome, Paris.

AYMARD, A.

(1967): “Les otages au début de l'empire", [en] Études d'Histoire Ancienne, Paris, 451460.

(1967a): "Philippe de Macédoine, otage à Thèbes", [en] Études d'Histoire Ancienne, Paris, 428-435.

(1967b): "Les otages carthaginois à la fin de la deuxième guerre punique", [en] Études d'Histoire Ancienne, Paris, 437-450.

BAdian, E. (1959): "Rome and Antiochus the Great: A Study in Cold War", Classical Philology 54/2, 81-99 (http://dx.doi.org/10.1086/364346).

Baldson, J. P. V. D. (1954): “Rome and Macedon, 205-200 B.C.”, JRS 44, 30-42 (http:// dx.doi.org/10.2307/297553).

BASTIEN, J.-L. (2007): Le Triomphe roman et son utilisation politique, Roma. 
Beard, M. (2009): El Triunfo romano. Una historia de Roma a través de la celebración de sus victorias, Barcelona.

Bellini, V. (1964): “Deditio in fidem”, RHDFE 42, 488-456.

Bickerman, E. J. (1932): “Bellum Antiochicum”, Hermes 67, 47-76.

Bonnefond-Coudry, M. (1989): Le Sénat de la République Romaine, de la Guerre d'Hannibal à Auguste, Roma.

BRISCOE, J.

(2003): A Commentary on Livy Books XXXIV-XXXVII, New York.

(2008): A Commentary on Livy Books 38-40, Oxford.

(2009): A Commentary on Livy Books XXXI-XXXIII, New York.

Broughton, R. S. (1951): The Magistrates of the Roman Republic I: 509-100 BC, New York.

Burton, P. J. (2011): Friendship and Empire. Roman Diplomacy and Imperialism in the Middle Republic (353-146 B.C.), Cambridge.

Churchill, J. B. (1999): “Ex qua quod vellent facerent: Roman Magistrate's Autorithy over Praeda and Manubiae", TAPhA 129, 85-116 (http://dx.doi.org/10.2307/284425).

Combès, R. (1966): Imperator (Recherches sur l'emploi et la signification du titre d'imperator dans la Rome républicaine), Paris.

Coudry, M. (2009): "Partage et gestión du butin dans la Rome républicaine: procédures et enjeux", [en] Coudry - Humm (eds.), 2009, Stuttgart, 21-80.

Coudry, M. - Humm, L. (EDS.), (2009): Praeda. Butin de guerre et société dans la Rome républicaine, Stuttgart.

Crawford, M. H. (1974): Roman Republican Coinage. II, Cambridge.

DAhlHeIm, W. (1965): Deditio und societas, München.

DELL, H.

(1967): "Origin and Nature of Illyrian Piracy", Historia: Zeitschrift für Alte Geschichte 16, 344-358.

(1970): "Demetrius of Pharus and the Istrian War", Historia: Zeitschrift für Alte Geschichte 19/1, 30-38.

Dyson, S. L. (1985): The Creation of the Roman Frontier, New Jersey.

Eckstein, A. M. (2008): Rome enters the Greek East. From Anarchy to Hierarchy in the Hellenistic Mediterranean, 230-170 BC, Malden-Oxford-Victoria.

Errington, R. M. (1989): "Rome against Philip and Antiochus", [en] A. E. Astin (ed.), The Cambridge Ancient History, Vol. VIII, Rome and the Mediterranean to 133 B.C., Cambridge, 244-289 (http://dx.doi.org/10.1017/CHOL9780521234481.009).

Fernández Canosa, J. A. (1988): "Rutura das hostilidades, alianza e paz, e fides na Hispania prerromana: instituzions de guerra", [en] Actas del I Congreso Peninsular de Historia Antigua (Santiago, 1985), Santiago, vol. 2, 239-248.

Flurl, W. (1969): Deditio in fidem: Untersuchungen zu Livius and Polybios, München.

Foraboschi, D. (1992): Lineamenti di storia della Cisalpina romana, Roma.

GARCÍA RiAZA, E.

(1999): "Las cláusulas económicas en las negociaciones de paz romano-celtibéricas", [en] F. Burillo Mozota (coord.), IV Simposio sobre Celtiberos. Economía. Homenaje a José Luis Argente Oliver (Daroca, Zaragoza, 25-27 septiembre 1997), Zaragoza, 515-520. 
(1999a): "Especie, metal, moneda: consideraciones en torno a la cuantificación de las exacciones romanas en Hispania republicana", [en] R. M. S. Centeno - Ma P. García Bellido - G. Mora (eds.), Rutas, ciudades y moneda en Hispania. Actas II Encontro Peninsular de Numismática Antiga (Porto, 18-21 março 1988), (=Anejos de AEspA 20), Madrid, 39-46. (1999b): "El cómputo del metal precioso en los botines de guerra hispano-republicanos", HAnt 23, 119-136.

(1999c): "La financiación de los ejércitos en época romano-republicana", [en] Moneda $i$ exèrcits. III Curs d'Història monetària d'Hispània, Barcelona, 39-58.

(2002): Celtíberos y lusitanos frente a Roma: diplomacia y derecho de guerra (=Anejos de Veleia, Series Minor 18), Vitoria-Gasteiz.

(2006): "Rehenes y diplomacia en la Hispania romano-republicana", [en] G. Bravo - R. González Salinero (eds.), Minorías y sectas en el mundo romano. Actas del III Coloquio de la AIER (=Signifer, Monografías y Estudios de Antigüedad Griega y Romana 20), Madrid, $17-33$.

(2007): “Tempus Poenae. Represalias contra poblaciones sometidas durante la expansión romana en Hispania", [en] G. Bravo - R. González Salinero (eds.), Formas y usos de la violencia en el mundo romano. Actas del IV Coloquio de la AIER (=Signifer, Monografías y Estudios de Antigüedad Griega y Romana 26), Madrid, 19-30.

(2011): "Derecho de Guerra en Occidente durante la expansión romano-republicana. Planteamientos metodológicos", [en] García Riaza (ed.), 2011, 31-66.

(2012): "Territorios indígenas y derecho de guerra romano en Hispania", [en] Santos Yanguas - Cruz Andreotti (eds.), 2012, 207-234.

García Riaza, E. (ED.), (2011): De fronteras a provincias. Interacción e integración en Occidente (ss. III-I a.C.), Palma de Mallorca.

GARrido-Hory, M. (1996): "Captifs et prisonniers de guerre dans les 18 premiers colloques du G.I.R.E.A.”, [en] Sánchez León - López Nadal (eds.), 1996, 141-164.

GonzÁlez Román, C. (1990): “Dediticii y clientes en el área ibérica de la Hispania republicana con anterioridad a las guerras civiles", [en] VV.AA., Esclavos y semilibres en la Antigüedad clásica, Madrid, 187-206.

Grainger, J. D.

(1995): “The Campaign of Cn. Manlius Vulso in Asia Minor”, Anatolian Studies 45, 23-42 (http://dx.doi.org/10.2307/3642912).

(2002): The Roman War of Antiochos the Great, Leiden-Boston.

Gruen, E. S. (1984): The Hellenistic World and the Coming of Rome, Berkeley-Los Angeles-London.

HARRIS, W. V.

(1971): “On War and Greed in the Second Century B.C.", AHR 76, 1371-1385 (http:// dx.doi.org/10.2307/1870513).

(1979): War and Imperialism in Republican Rome, 327-70 BC, Oxford.

Heuss, A. (1971): Römische Geschichte, Braunschweig.

KNAPP, R. C. (1977): Aspects of the Roman Experience in Iberia 206-100 B.C., Valladolid.

LAmboglia, N. (1932): La prima fase delle guerre romano-liguri 238-230 a.C. (=Collana storico-archaeologica della Liguria occidentale, I, 6), Imperia.

LONIS, R.

(1969): Les usages de la Guerre entre Grecs et Barbares des guerres médiques au milieu du IVe siècle avant Jésus Christ, Paris. 
(1977): "Les otages dans les relations internationales en Grèce classique”, [en] Mélanges offerts à Léopold Sédar Senghor, Dakar, 215-234.

López Melero, R. - SÁnchez Abal, J. L. - García Jiménez, S. (1984): "El bronce de Alcántara: una deditio del 104 a.C.", Gerión 2, 265-323.

Marco Simón, F. (2006): “Intimidación y terror en la época de las Guerras Celtibéricas”, [en] G. Urso (ed.), Terror et Pavor. Violenza, Intimidazione, Clasdentinità del mondo antico. Atti del Convegno Internazionale (Cividale del Friuli, 22-24 settembre 2005), Pisa, 197213.

Martínez Gázquez, J. (1992): La campaña de Catón en Hispania, Barcelona.

Martínez Morcillo, J. A.

(2011): "La contravención del ius belli durante la primera mitad del siglo II a.C.: cinco casos de estudio", [en] García Riaza (ed.), 2011, 67-79.

(2012): "Lucio Emilio Paulo y el derecho de guerra", SHHA 30, 271-292.

(2012a): "Acabar con la identidad del enemigo: política romana de reorganización de territorios sometidos en contexto de guerra (s. II a.C.)", [en] J. M. Aldea Celada - P. Ortega Martínez - I. Pérez Miranda - Ma . R. de Soto García (coords.), Historia, Identidad y Alteridad. Actas del IIIer Congreso Interdisciplinar de Jóvenes Historiadores (=Temas y Perspectivas de la Historia 2), Salamanca, 137-161.

(2013): "El asalto de núcleos de población: bases jurídicas, procedimiento y consecuencias durante la República romana", ETF $H^{a}$ Ant. 26, 107-122 (http://dx.doi.org/10.5944/ etfii.26.2013.13740).

Meloni, P. (1953): Perseo e la fine della monarchia macedone, Roma.

Moscovich, M. J. (1983): "Hostage princes and Roman Imperialism in the second century BC", EMC 27/2, 297-309.

NDIAYE, S. (1995): “Le recours aux otages à Rome sous la République”, DHA 21, 149-165 (http://dx.doi.org/10.3406/dha.1995.2223).

NicosiA, G. (1996): "Prigiona di guerra e perdita della libertà nell'esperienza giuridica romana”, [en] Sánchez León - López Nadal (eds.), 1996, 39-48.

NACO, T.

(2001): "Milites in oppidis hibernabant. El hospitium militare invernal en ciudades peregrinas y los abusos de la hospitalidad sub tectis durante la República", DHA 27, 63-90 (http://dx.doi.org/10.3406/dha.2001.2449).

(2003): Vectigal incertum. Economía de guerra y fiscalidad republicana en el occidente romano: su impacto histórico en el territorio (218-133 a.C.), (=BAR International Series 1158), Oxford.

(2005): "Vectigal Incertum. Guerra y fiscalidad republicana en el siglo II a. de C.", Klio 87/2, 366-395.

(2006): "Una historia de la primera fase de la intervención romana en Hispania (218-133 a.C.)", [en] Naco - Arrayás (eds.), 2006, 81-102.

(2010): "The Republican War Economy Strikes Back: a "minimalist” approach", [en] F. Kirbihler - N. Barrandón (eds.), Administrer les provinces de la République romaine 1, Rennes, 171-180.

ÑACO, T. - ARRAYÁs, I. (EDS.), (2006): War and Territory in the Roman World (=BAR International Series 1538), Oxford. 
Olesti-Vila, O. (2006): "El control de los territorios del Nordeste Peninsular (218-100 a.C.): un modelo a debate", [en] Ñaco - Arrayás (eds.), 2006, 119-148.

Panagopoulos, A. (1978): Captives and Hostages in the Peloponnesian war, Athens.

PARADISI, B. (1941): Deditio in fidem, Milano (Ex: Studi di Storia e diritto in honore di Arrigo Solmi, vol. 1).

Pérez Rubio, A. - Sánchez Moreno, E. - Per Gimeno, L. - Martínez Morcillo, J. A. García RiazA, E. (2013): "Symmachíai celtibéricas (220-133 a.C.): coaliciones militares en el horizonte del Imperialismo Mediterráneo", [en] Acta Palaeohispanica XI. Actas del XI Coloquio Internacional de lenguas y culturas prerromanas de la península ibérica, Valencia, 24-27 de octubre de 2012 (=Palaeohispanica 13), Zaragoza, 675-697.

Peyre, C. (1979): La Cisalpine gauloise du IIIé au Iér siècle avant J.-C., Paris.

Pina Polo, F.

(2004): "Deportaciones como castigo e instrumento de colonización durante la República Romana. El caso de Hispania" [en] F. Marco - F. Pina Polo - J. Remesal (eds.), Vivir en tierra extraña: emigración e integración cultural en el mundo antiguo (=Col.lecció Instrumenta 16), Barcelona, 211-246.

(2006): "Deportation, Kolonisation, Migration: Bevölkerungsverschiebungen im republikanischen Italien und Formen der Identitätsbildung”, [en] M. Jehne - R. Pfeilschifter (Hgg.), Herrschaft ohne Integration? Rom und Italien in republikanischer Zeit (=Studien zur Alten Geschichte 4), Frankfurt am Main, 171-206.

Rosenstein, N. (2012): Rome and the Mediterranean 290 to 146 BC. The Imperial Republic, Edinburgh.

Richardson, J. S. (1986): Hispaniae. Spain and the Development of Roman Imperialism, 218-82 BC, Cambridge.

SALINAS, M.

(1986): Conquista y romanización de Celtiberia, Salamanca.

(1989): “Quintus Fulvius Q. F. Flaccus”, SHHA 7, 67-84.

SÁnchez León, Ma L. - LóPez Nadal, G. (eds.), (1996): Captius i Esclaus a l'Antiguitat $i$ al món modern. Actes del XIX Col-loqui Internacional del GIREA (Palma de Mallorca, 2-5 octubre 1995), (=Diáphora Series 7), Napoli.

Santos Yanguas, J. - CRuz Andreotti, G. (EDS.), (2012): Romanización, fronteras y etnias en la Roma antigua: el caso hispano (=Anejos de Veleia, Acta 12, Revisiones de Historia Antigua VII), Vitoria-Gasteiz.

SCULlard, H. H.

(1945): "Charops and Roman Policy in Epirus", JRS 35, 58-64 (http://dx.doi. org/10.2307/297278).

(1973): Roman Politics, 220-150 BC, London.

Serrano, J. M. (1989): "Documentos adicionales relativos a la amicitia”, Habis 20, 175-184.

ShatzMan, I. (1972): “The Roman General's Authority over Booty”, Historia 21, 177-205.

TARPIn, M. (2009): “Les manubiae dans la procédure d'appropiatin du botin”, [en] Coudry - Humm (eds.), 2009, 81-102.

TAÜBLER, E. (1915): Imperium Romanum: Studien zur Entwickelunggsgeschichte des romischen Reichs, I, Berlin. 
Versnel, H. S. (1970): Triumphus. An Inquiry into the Origin, Development and Meaning of the Roman Triumph, Leiden.

VissCHER, F. (1946): "La deditio internationale et l'affaire des Fourches Caudines", CRAI, 82-95 (http://dx.doi.org/10.3406/crai.1946.77941).

Volkmann, H. (1961): Die Massenversklavungen der Einwohner eroberter Städte in der hellenistich-römischen Zeit, Wiesbaden.

ZIEGLER, K. H. (1991): “Deditio und fides im römischen Völkerrecht”, ZRG 108, 279-285.

Ziolkowski, A. (1995): "Urbs direpta or how the Romans sacked cities", [en] J. Rich - G. Shipley (eds.), War and Society in the Roman World (=Leicester-Nottingham Studies in Ancient World 5), London-New York, 69-91. 